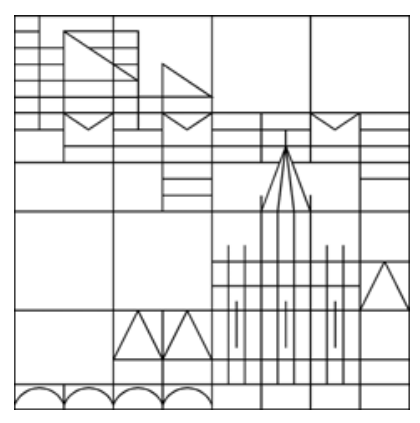

Pseudodifferenzial operators with non-regular operator-valued symbols

\author{
Bienvenido Barraza Martínez \\ Robert Denk \\ Jairo Hernández Monzón
}

Konstanzer Schriften in Mathematik

Nr. 307, September 2012

ISSN 1430-3558

Konstanzer Online-Publikations-System (KOPS)

URL: http://nbn-resolving.de/urn:nbn:de:bsz:352-202597 



\title{
PSEUDODIFFERENTIAL OPERATORS WITH NON-REGULAR OPERATOR-VALUED SYMBOLS
}

\author{
BIENVENIDO BARRAZA MARTÍNEZ, ROBERT DENK, \\ AND JAIRO HERNÁNDEZ MONZÓN
}

\begin{abstract}
In this paper, we consider pseudodifferential operators with operatorvalued symbols and their mapping properties, without assumptions on the underlying Banach space $E$. We show that, under suitable parabolicity assumptions, the $W_{p}^{k}\left(\mathbb{R}^{n}, E\right)$-realization of the operator generates an analytic semigroup. An application to non-autonomous pseudodifferential Cauchy problems gives the existence and uniqueness of a classical solution. Our approach is based on oscillatory integrals and kernel estimates for them.
\end{abstract}

\section{INTRODUCTION}

In this paper, we consider pseudodifferential operators with operator-valued symbols, their mapping properties, generation of an analytic semigroup and the application to non-autonomous vector-valued evolution equations. Operator-valued symbols and vector-valued function spaces appear in a natural way in several applications, e.g. in models of coagulation-fragmentation processes where an additional parameter (the cluster size) appears in the model, see [Am00]. Another example is given by boundary value problems in cylindrical domains which can be treated by a Fourier multiplier approach which leads to operator-valued symbols, too (see [NS11]). Therefore, during the last decade, the investigation of vector-valued function spaces and differential equations with operator-valued coefficients has gained increasing interest.

Our work is motivated by two directions of research: On one hand, operatorvalued Fourier multipliers in arbitrary Banach spaces and their mapping properties have been considered in [Am97]. Based on these Fourier multiplier results, the generation of an analytic semigroup for differential operators could be shown in [Am01]. On the other hand, under an additional geometric assumption on the Banach space (to be a UMD space), Weis could establish in [We01] a vector-valued Mikhlin type theorem (see also [NNH02], [GW03]). This was the basis for a large number of results on maximal regularity for differential and pseudodifferential operators in UMD spaces. As references for operator-valued differential boundary value problems in UMD spaces and the connection to $\mathcal{R}$-sectoriality and maximal regularity, we mention [DHP03] and [KW04]. For vector-valued pseudodifferential operators in UMD spaces, we refer to [DK07], [PS06] and the references therein.

Date: August 31, 2012.

2010 Mathematics Subject Classification. 35S05, 47D06, 35R20.

Key words and phrases. Pseudodifferential operators, operator-valued symbols, generation of analytic semigroup. 
The restriction to UMD spaces, however, excludes natural state spaces as $L^{1}$, and therefore the present paper deals with pseudodifferential operators with operatorvalued symbols in arbitrary Banach spaces. We consider operator-valued symbols $a$ in the standard Hörmander class with limited smoothness both in the variable $x$ and in the covariable $\xi$ and with positive order $m>0$. Similar operators were also considered in [Ki01],[Ki03] with additional assumptions on the symbol (e.g., order $m>1$, existence of a homogeneous principal part, infinite smoothness in $\xi$ ).

One of the main results in the present paper states that, under suitable parabolicity assumptions, the $W_{p}^{k}\left(\mathbb{R}^{n}, E\right)$-realization of the symbol $a$ generates an analytic semigroup in $W_{p}^{k}\left(\mathbb{R}^{n}, E\right)$, see Theorem 4.2 and Corollary 4.3 below. An application to non-autonomous pseudodifferential Cauchy problems gives the existence and uniqueness of a classical solution (Theorem 5.3). Our approach is based on oscillatory integrals and careful kernel estimates for them, the technical key result being Lemma 3.2. For the case of constant coefficients, results on vector-valued pseudodifferential operators were obtained in our paper [BDH12].

\section{VECTOR-VALUED PSEUDO-DIFFERENTIAL OPERATORS}

In the following, we set $|x|:=\left(\sum_{j=1}^{n}\left|x_{j}\right|^{2}\right)^{1 / 2},|x, \mu|:=\left(|x|^{2}+\mu^{2}\right)^{1 / 2},\langle x\rangle:=$ $\left(1+|x|^{2}\right)^{1 / 2}$, and $\langle x, \mu\rangle:=\left(1+|x|^{2}+\mu^{2}\right)^{1 / 2}$ for $x=\left(x_{1}, \ldots, x_{n}\right) \in \mathbb{R}^{n}$ and $\mu \in \mathbb{R}$. Throughout this paper, $(E,\|\cdot\|)$ denotes an arbitrary Banach space, and for locally convex spaces $X, Y$ we write $L(X, Y)$ for the space of all continuous linear operators from $X$ to $Y$, and set $L(X):=L(X, X)$. Let $C_{b}^{\infty}\left(\mathbb{R}^{n}, E\right)$ be the space of all $u: \mathbb{R}^{n} \rightarrow E$ such that $\partial^{\alpha} u$ is bounded and continuous for all $\alpha \in \mathbb{N}_{0}^{n}$ where we use standard multi-index notation. The space $C_{b}^{\infty}\left(\mathbb{R}^{n}, E\right)$ is endowed with the locally convex topology given by the seminorms $\|u\|_{k}:=\max _{|\alpha| \leq k} \sup _{x \in \mathbb{R}^{n}}\left\|\partial^{\alpha} u(x)\right\|_{E}$, $k \in \mathbb{N}_{0}$. The Schwartz space of rapidly decreasing $E$-valued functions is denoted by $\mathscr{S}\left(\mathbb{R}^{n}, E\right)$, and for $k \in \mathbb{N}_{0}$ and $1 \leq p<\infty$, we write $W_{p}^{k}\left(\mathbb{R}^{n}, E\right)$ for the $E$-valued Sobolev space endowed with the norm

$$
\|u\|_{W_{p}^{k}\left(\mathbb{R}^{n}, E\right)}:=\left(\sum_{|\alpha| \leq k}\left\|\partial^{\alpha} u\right\|_{L^{p}\left(\mathbb{R}^{n}, E\right)}^{p}\right)^{1 / p} .
$$

Here $\|\cdot\|_{L^{p}\left(\mathbb{R}^{n}, E\right)}$ stands for the norm in the Lebesgue-Bochner space $L^{p}\left(\mathbb{R}^{n}, E\right)$. We set $\overline{\mathbb{R}_{+}^{n}}:=\mathbb{R}^{n} \times[0, \infty), D^{\alpha}:=(-i)^{|\alpha|} \partial^{\alpha}$ and $d(\xi, y):=(2 \pi)^{-n} d(\xi, y)$.

We start with the definition of the symbol class (which is a non-smooth parameterdependent version of the standard Hörmander class $S_{1,0}^{m}$ ) and the related pseudodifferential operators. In the following, for $n \in \mathbb{N}$ and $m \in \mathbb{R}$, we set $\rho_{n}:=$ $\left\{\begin{array}{ll}n+1, & \text { if } n \text { is odd, } \\ n+2, & \text { if } n \text { is even. }\end{array}\right.$ and $\rho_{n, m}:= \begin{cases}{[n+m]+1,} & \text { if }[n+m] \text { is odd, } \\ {[n+m]+2,} & \text { if }[n+m] \text { is even. }\end{cases}$

Definition 2.1. Let $m \in \mathbb{R}, \nu \in[0, \infty]$, and $r \in \mathbb{N}_{0}$.

a) We define $S^{m, \nu, r}:=S^{m, \nu, r}\left(\mathbb{R}_{x}^{n} \times \overline{\mathbb{R}_{+}^{n}}, L(E)\right)$ as the set of all functions $a: \mathbb{R}_{x}^{n} \times$ $\overline{\mathbb{R}_{+}^{n}} \rightarrow L(E)$ such that

(i) $x \mapsto a(x, \xi, \mu) \in C^{r}\left(\mathbb{R}^{n}, L(E)\right)$ for all $(\xi, \mu) \in \overline{\mathbb{R}_{+}^{n}}$,

(ii) $(\xi, \mu) \mapsto a(x, \xi, \mu) \in C^{[\nu]}\left(\overline{\mathbb{R}_{+}^{n}}, L(E)\right)$ for all $x \in \mathbb{R}^{n}$, and

(iii) $|a|_{m}^{(\nu, r)}<\infty$ where

$$
|a|_{m}^{(\nu, r)}:=\sup _{x \in \mathbb{R}^{n}} \sup _{(\xi, \mu) \in \overline{\mathbb{R}_{+}^{n}}} \max _{|\alpha|+k \leq \nu} \max _{|\beta| \leq r}\langle\xi, \mu\rangle^{-m+|\alpha|+k}\left\|\partial_{x}^{\beta} \partial_{\xi}^{\alpha} \partial_{\mu}^{k} a(x, \xi, \mu)\right\|_{L(E)} .
$$


b) For $a \in S^{m, \nu, r}$ with $\nu \geq \rho_{n}$, the pseudo-differential operator $\operatorname{op}(a):=a(x, D, \mu)$ is defined by

$$
[a(x, D, \mu) u](x):=\mathrm{Os}-\iint_{\mathbb{R}^{2 n}} e^{i \xi \cdot y} a(x, \xi, \mu) u(x-y) d(\xi, y) \quad\left(x \in \mathbb{R}^{n}\right)
$$

for $u \in C_{b}^{\infty}\left(\mathbb{R}_{x}^{n}, E\right)$.

For the convenience of the reader, we state the definition and some properties of the oscillatory integral in the appendix.

In the following, we will also study parameter-dependent double symbols $a=$ $a(x, y, \xi, \mu)$ with $a \in S^{m, \nu, r}\left(\mathbb{R}_{x, y}^{2 n} \times \overline{\mathbb{R}_{+}^{n}}, L(E)\right)$ with $\nu \geq \rho_{n}$ and $r>\rho_{n, m}$. Here we set

$$
[a(x, y, D, \mu) u](x):=\mathrm{Os}-\iint_{\mathbb{R}^{2 n}} e^{i \xi \cdot y} a(x, x-y, \xi, \mu) u(x-y) d(\xi, y) \quad\left(x \in \mathbb{R}^{n}\right)
$$

for $u \in C_{b}^{\infty}\left(\mathbb{R}^{n}, E\right)$. In the particular case where $a(x, y, \xi, \mu)$ does not depend on $x$ (dual symbol), we will write $a(y, D, \mu)$.

Remark 2.2. a) For $\nu \geq \rho_{n}$, Lemma A.4 shows that the oscillatory integral in $b$ ) exists and due to Lemma $A .5$ too, that $a(x, D, \mu) \in L\left(C_{b}^{\infty}\left(\mathbb{R}^{n}, E\right), C_{b}^{r}\left(\mathbb{R}^{n}, E\right)\right)$ for every fixed $\mu \in[0, \infty)$.

b) For $\nu \geq \rho_{n}$ and $r>\rho_{n, m}$, the oscillatory integral in (1) exists. Note that for $n+m \leq 0$ the condition $r>\rho_{n, m}$ is satisfied for all $r \in \mathbb{N}$.

The following result is taken from [Ki03], Remark 2.2.6.

Lemma 2.3. Let $a_{i} \in S^{m_{i}, \nu, r}, i=1,2$, with $m_{1}, m_{2} \in \mathbb{R}, \nu \geq \rho_{n}$ and $r \geq$ $\rho_{2 n, m_{1}+m_{2}}$. Then $a_{1}(x, D, \mu) a_{2}(y, D, \mu)=\operatorname{op}\left(a_{1}(x, \xi, \mu) a_{2}(y, \xi, \mu)\right)$.

Definition 2.4. a) Let $m, \nu \in(0, \infty)$ and $r \in \mathbb{N}_{0}$, and let $a \in S^{m, \nu, r}$. Then $a$ is called parameter-elliptic if there are constants $\kappa>0$ and $\omega \geq 0$ such that for all $(x, \xi, \mu) \in \mathbb{R}^{n} \times \mathbb{R}^{n} \times[0, \infty)$ with $|\xi, \mu| \geq \omega$ we have

(i) $a(x, \xi, \mu): E \rightarrow E$ is bijective, and

(ii) $\left\|a(x, \xi, \mu)^{-1}\right\|_{L(E)} \leq \kappa\langle\xi, \mu\rangle^{-m}$.

The set of all $a \in S^{m, \nu, r}$ satisfying (i)-(ii) will be denoted by $E_{\kappa, \omega}^{m, \nu, r}:=E_{\kappa, \omega}^{m, \nu, r}\left(\mathbb{R}_{x}^{n} \times\right.$ $\left.\overline{\mathbb{R}_{+}^{n}}, L(E)\right)$.

b) Let $m \in(0, \infty)$ and $r, \nu \in \mathbb{N}_{0}$, and let $a \in S^{m, \nu, r}\left(\mathbb{R}_{x}^{n} \times \mathbb{R}_{\xi}^{n}, L(E)\right)$ be a symbol independent of $\mu \in(0, \infty)$. Then $a$ is called parabolic with constants $\kappa>0, \omega \geq 0$ if $(x, \xi, \mu) \mapsto a(x, \xi)+\mu^{m} e^{i \theta}$ belongs to $E_{\kappa, \omega}^{m, \nu, r}$ for all $|\theta| \leq \frac{\pi}{2}$.

For a symbol $a \in E_{\kappa, \omega}^{m, \nu, r}$, we define a smooth version of the inverse symbol in the following way: Let $\psi \in C^{\infty}\left(\mathbb{R}^{n+1}\right)$ with $0 \leq \psi \leq 1, \psi(\xi, \mu)=0$ for $|\xi, \mu| \leq \frac{1}{2}$ and $\psi(\xi, \mu)=1$ for $|\xi, \mu| \geq 1$ be a zero extinction function. For $\omega_{0}>\omega$ we set

$$
a^{\#}(x, \xi, \mu):= \begin{cases}\psi\left(\frac{\xi}{2 \omega_{0}}, \frac{\mu}{2 \omega_{0}}\right) a(x, \xi, \mu)^{-1}, & \text { if } x \in \mathbb{R}^{n},|\xi, \mu| \geq \omega_{0}, \\ 0, & \text { if } x \in \mathbb{R}^{n},|\xi, \mu| \leq \omega_{0}\end{cases}
$$

Then $a^{\#}(x, \xi, \mu)=0$ if $|\xi, \mu| \leq \omega_{0}$ and $a^{\#}(x, \xi, \mu)=a(x, \xi, \mu)^{-1}$ if $|\xi, \mu| \geq 2 \omega_{0}$. 


\section{KEY ESTIMATES FOR PARAMETER-ELLIPTIC SYMBOLS}

In this section, we will prove key estimates for the inverse of parameter-elliptic symbols and mapping properties for the corresponding operators. We start with a result which shows that the inverse of a parameter-elliptic symbol is again in the calculus.

Lemma 3.1. Let $m, \nu \in(0, \infty), r \in \mathbb{N}_{0}$, and let $\mathcal{A} \subset S^{m, \nu, r}$ be bounded, i.e. there exists a constant $K>0$ such that $|a|_{m}^{(\nu, r)} \leq K(a \in \mathcal{A})$. Assume moreover that $\mathcal{A} \subset E_{\kappa, \omega}^{m, \nu, r}$. Then there exists a constant $C=C(K, \kappa, \nu, r)>0$ such that

$$
\left\|\partial_{x}^{\beta} \partial_{\xi}^{\alpha} \partial_{\mu}^{k} a^{\#}(x, \xi, \mu)\right\|_{L(E)} \leq C\langle\xi, \mu\rangle^{-m-|\alpha|-k}
$$

for all $x \in \mathbb{R}^{n},(\xi, \mu) \in \overline{\mathbb{R}_{+}^{n}},|\alpha|+k \leq \nu,|\beta| \leq r$, and $a \in \mathcal{A}$, i.e., $\left\{a^{\#}: a \in \mathcal{A}\right\} \subset$ $S^{-m, \nu, r}$ is bounded.

Proof. Note that $\left\{\langle\xi, \mu\rangle^{m+|\alpha|+k}\left\|\partial_{x}^{\beta} \partial_{\xi}^{\alpha} \partial_{\mu}^{k} a^{\#}(x, \xi, \mu)\right\|_{L(E)}: x \in \mathbb{R}^{n},|\xi, \mu| \leq 2 \omega_{0},|\alpha|+\right.$ $k \leq \nu,|\beta| \leq r\}$ is bounded by continuity and compactness. Therefore, it suffices to consider the case $|\xi, \mu| \geq 2 \omega_{0}$.

Let $x \in \mathbb{R}^{n},|\xi, \mu| \geq 2 \omega_{0}, \alpha, \beta \in \mathbb{N}_{0}^{n} \backslash\{0\}, k \in \mathbb{N}_{0}$ with $|\beta| \leq r$ and $|\alpha|+k \leq \nu$. Then $\partial_{x}^{\beta} \partial_{\xi}^{\alpha} \partial_{\mu}^{k} a(x, \xi, \mu)^{-1}$ is a finite linear combination of terms of the form

$$
\begin{gathered}
a(x, \xi, \mu)^{-1}\left[\partial_{x}^{\beta^{(1)}} \partial_{\xi}^{\alpha^{(1)}} \partial_{\mu}^{k^{(1)}} a(x, \xi, \mu)\right] a(x, \xi, \mu)^{-1} \ldots \\
\ldots\left[\partial_{x}^{\beta^{(p)}} \partial_{\xi}^{\alpha^{(p)}} \partial_{\mu}^{k^{(p)}} a(x, \xi, \mu)\right] a(x, \xi, \mu)^{-1}
\end{gathered}
$$

with $1 \leq p \leq|\alpha|+|\beta|+k$ and with $\alpha^{(i)}, \beta^{(i)} \in \mathbb{N}_{0}^{n}, k^{(i)} \in \mathbb{N}(i=1, \ldots, p)$ satisfying $\sum_{i=1}^{p} \alpha^{(i)}=\alpha, \sum_{i=1}^{p} \beta^{(i)}=\beta, \sum_{i=1}^{p} k^{(i)}=k$. The norm of the operator in (2) can be estimated by

$$
\begin{aligned}
\left\|a(x, \xi, \mu)^{-1}\right\|_{L(E)}^{p+1} & \prod_{i=1}^{p}\left\|\partial_{x}^{\beta^{(i)}} \partial_{\xi}^{\alpha^{(i)}} \partial_{\mu}^{k^{(i)}} a(x, \xi, \mu)\right\|_{L(E)} \\
& \leq \kappa^{p+1}\langle\xi, \mu\rangle^{-m(p+1)} \prod_{i=1}^{p}|a|_{m}^{(\nu, r)}\langle\xi, \mu\rangle^{m-\left|\alpha^{(i)}\right|-k^{(i)}} \\
& =\kappa^{p+1}\left(|a|_{m}^{(\nu, r)}\right)^{p}\langle\xi, \mu\rangle^{-m-|\alpha|-k} .
\end{aligned}
$$

Summing up over all terms of the form (2), we obtain

$$
\left\|\partial_{x}^{\beta} \partial_{\xi}^{\alpha} \partial_{\mu}^{k} a^{-1}(x, \xi, \mu)\right\|_{L(E)} \leq C\langle\xi, \mu\rangle^{-m-|\alpha|-k}
$$

with a constant $C=C(K, \kappa, \nu, r)$.

From now on we set $\chi_{\varepsilon}(\xi, y):=\chi(\varepsilon \xi) \chi(\varepsilon y)$ for $\varepsilon>0$ and $\xi, y \in \mathbb{R}^{n}$, where $\chi \in \mathscr{S}\left(\mathbb{R}^{n}\right)$ with $\chi(0)=1$. The following result is the key estimate for parameterdependent symbols of negative order.

Lemma 3.2. Let $b \in S^{-m, \nu, r}$ with $m>0, \nu \geq n+1, r \in \mathbb{N}_{0}$ and $\omega_{0}>0$. For $\varepsilon \in(0,1)$ define

$$
K_{\varepsilon}(x, y, \mu):=\int_{\mathbb{R}^{n}} e^{i \xi \cdot y} \chi_{\varepsilon}(\xi, y) b(x-y, \xi, \mu) d \xi \quad\left((x, y, \mu) \in \mathbb{R}^{2 n} \times\left[\omega_{0}, \infty\right)\right) .
$$

a) There exists a constant $C$ such that

$$
\left\|K_{\varepsilon}(x, y, \mu)\right\|_{L(E)} \leq C|b|_{-m}^{(\nu, r)} \mu^{-m+n} \frac{|\mu y|^{\theta_{0}}+|\mu y|^{\theta_{1}}}{|\mu y|^{n}(1+|\mu y|)} \quad\left((x, y, \mu) \in \mathbb{R}^{2 n} \times\left[\omega_{0}, \infty\right)\right)
$$


where $\theta_{0}:=\frac{1}{2} \min \{m, 1\}$ and $\theta_{1}:=\frac{1}{2}$. Moreover,

$$
\left\|K_{\varepsilon}(x, \cdot, \mu)\right\|_{L^{1}\left(\mathbb{R}^{n}, L(E)\right)} \leq C|b|_{-m}^{(\nu, r)} \mu^{-m} \quad\left((x, \mu) \in \mathbb{R}^{n} \times\left[\omega_{0}, \infty\right)\right)
$$

holds with a constant $C$ independent of $\varepsilon, x$ and $\mu$.

b) There exists a strongly measurable function $K: \mathbb{R}^{2 n} \times\left[\omega_{0}, \infty\right) \rightarrow L(E)$ with $K_{\varepsilon}(x, y, \mu) \rightarrow K(x, y, \mu)(\varepsilon \searrow 0)$ pointwise, and the estimate (3) holds with $K_{\varepsilon}$ being replaced by $K$.

Proof. a) Substituting $\xi \mapsto \mu \xi$, we have

$$
K_{\varepsilon}(x, y, \mu)=\mu^{n} \int_{\mathbb{R}^{n}} e^{i \mu \xi \cdot y} \chi_{\varepsilon}(\mu \xi, y) b(x-y, \mu \xi, \mu) d \xi .
$$

We fix $\gamma \in \mathbb{N}_{0}^{n}$ with $|\gamma|=n+i, i \in\{0,1\}$. By assumption, $b(x-y, \cdot, \mu) \in$ $C^{|\gamma|}\left(\mathbb{R}^{n}, L(E)\right)$ and therefore

$$
\int_{\mathbb{R}^{n}} D_{\xi}^{\gamma}\left[\chi_{\varepsilon}(\mu \xi, y) b(x-y, \mu \xi, \mu)\right] d \xi=0
$$

With this and partial integration, we see

$$
(\mu y)^{\gamma} K_{\varepsilon}(x, y, \mu)=\mu^{n} \int_{\mathbb{R}^{n}}\left(e^{i \mu \xi \cdot y}-1\right) D_{\xi}^{\gamma}\left[\chi_{\varepsilon}(\mu \xi, y) b(x-y, \mu \xi, \mu)\right] d \xi
$$

To estimate the integrand, we apply the Leibniz rule noting that for $\alpha+\beta=\gamma$ we have

$$
\left|D_{\xi}^{\alpha} \chi_{\varepsilon}(\mu \xi, y)\right|=\left|D_{\xi}^{\alpha} \chi(\varepsilon \mu \xi)\right||\chi(\varepsilon y)| \leq c_{\alpha} \mu^{|\alpha|}\langle\mu \xi\rangle^{-|\alpha|}
$$

by Lemma A.3. This and

$$
\begin{aligned}
\left\|D_{\xi}^{\beta}[b(x-y, \mu \xi, \mu)]\right\|_{L(E)} & =\mu^{|\beta|}\left\|\left(D_{\xi}^{\beta} b\right)(x-y, \mu \xi, \mu)\right\|_{L(E)} \\
& \leq \mu^{|\beta|}|b|_{-m}^{(\nu, r)}\langle\mu \xi, \mu\rangle^{-m-|\beta|}
\end{aligned}
$$

gives

$$
\begin{aligned}
& \| D_{\xi}^{\gamma}\left[\chi_{\varepsilon}(\mu \xi, y)\right.b(x-y, \mu \xi, \mu)] \|_{L(E)} \\
& \leq \sum_{\alpha+\beta=\gamma} c_{\alpha \beta} \mu^{|\alpha|}\langle\mu \xi\rangle^{-|\alpha|}|\mu|^{|\beta|}\langle\mu \xi, \mu\rangle^{-m-|\beta|}|b|_{-m}^{(\nu, r)} \\
& \leq c_{\gamma} \mu^{|\gamma|}\langle\mu \xi\rangle^{-m-|\gamma|}|b|_{-m}^{(\nu, r)}
\end{aligned}
$$

where we used $\langle\mu \xi, \mu\rangle^{-m-|\beta|} \leq\langle\mu \xi\rangle^{-m-|\beta|}$. Now we apply the elementary estimate $\left|e^{i \mu \xi y}-1\right| \leq 2|\mu y|^{\theta}|\xi|^{\theta}$ valid for all $\theta \in(0,1)$ and obtain

$$
\begin{aligned}
\left\|(\mu y)^{\gamma} K_{\varepsilon}(x, y, \mu)\right\|_{L(E)} & \leq C \mu^{n+|\gamma|}|\mu y|^{\theta}|b|_{-m}^{(\nu, r)} \int_{\mathbb{R}^{n}}|\xi|^{\theta}\langle\mu \xi\rangle^{-m-|\gamma|} d \xi \\
& =C \mu^{n-m}|\mu y|^{\theta}|b|_{-m}^{(\nu, r)} I(\theta,|\gamma|)
\end{aligned}
$$

with $I(\theta,|\gamma|):=\int_{\mathbb{R}^{n}}|\xi|^{\theta}\left(\mu^{-2}+|\xi|^{2}\right)^{-m / 2-|\gamma| / 2} d \xi$.

For $|\gamma|=n+i$ with $i \in\{0,1\}$ and $\theta_{0}:=\frac{1}{2} \min \{m, 1\}, \theta_{1}:=\frac{1}{2}$, we have $\theta_{i} \in(0,1)$ and

$$
\begin{aligned}
& I\left(\theta_{i},|\gamma|\right)=\int_{|\xi| \leq 1}|\xi|^{\theta_{i}}\left(\mu^{-2}+|\xi|^{2}\right)^{-m / 2-|\gamma| / 2} d \xi \\
&+\int_{|\xi| \geq 1}|\xi|^{\theta_{i}}\left(\mu^{-2}+|\xi|^{2}\right)^{-m / 2-|\gamma| / 2} d \xi
\end{aligned}
$$




$$
\leq \omega_{0}^{-m-|\gamma|} \int_{|\xi| \leq 1}|\xi|^{\theta_{i}} d \xi+\int_{|\xi| \geq 1}|\xi|^{\theta_{i}-m-|\gamma|} d \xi \leq C<\infty
$$

due to $\theta_{i}>0$ and $\theta_{i}-m-|\gamma|<-n$. Therefore, for all $\gamma$ with $|\gamma|=n+i, i \in\{0,1\}$, we have

$$
\left\|(\mu y)^{\gamma} K_{\varepsilon}(x, y, \mu)\right\|_{L(E)} \leq C \mu^{n-m}|\mu y|^{\theta_{i}}|b|_{-m}^{(\nu, r)}
$$

and consequently

$$
\begin{aligned}
|\mu y|^{n+i}\left\|K_{\varepsilon}(x, y, \mu)\right\|_{L(E)} & \leq n^{(n+i) / 2} \sum_{|\gamma|=n+i}\left\|(\mu y)^{\gamma} K_{\varepsilon}(x, y, \mu)\right\|_{L(E)} \\
& \leq C \mu^{n-m}|\mu y|^{\theta_{i}}|b|_{-m}^{(\nu, r)} .
\end{aligned}
$$

Summing up these inequalities for $i=0$ and $i=1$, we obtain the first assertion in a). Since the function

$$
y \mapsto \mu^{n} \frac{|\mu y|^{\theta_{0}}+|\mu y|^{\theta_{1}}}{|\mu y|^{n}(1+|\mu y|)}
$$

belongs to $L^{1}\left(\mathbb{R}^{n}\right)$ and its $L^{1}$-norm does not depend on $\mu$, we obtain inequality (3).

b) Let $\varepsilon, \varepsilon^{\prime} \in(0,1), \xi, y \in \mathbb{R}^{n}$ and $\mu \in\left[\omega_{0}, \infty\right)$. From the proof of a) we see that

$$
\begin{aligned}
(\mu y)^{\gamma} & \left(K_{\varepsilon}(x, y, \mu)-K_{\varepsilon^{\prime}}(x, y, \mu)\right. \\
& \left.=\mu^{n} \int_{\mathbb{R}^{n}}\left(e^{i \mu \xi \cdot y}-1\right) D_{\xi}^{\gamma}\left[\chi_{\varepsilon}(\mu \xi, y)-\chi_{\varepsilon^{\prime}}(\mu \xi, y)\right] b(x-y, \mu \xi, \mu)\right] d \xi .
\end{aligned}
$$

From Lemma A.3 we know that $D_{\xi}^{\alpha}\left(\chi_{\varepsilon}(\mu \xi, y)-\chi_{\varepsilon^{\prime}}(\mu \xi, y)\right) \rightarrow 0$ for $\varepsilon, \varepsilon^{\prime} \searrow 0$ for all $\alpha \in \mathbb{N}_{0}^{n}$ and all $\mu, \xi, y$. Therefore the integrand in (5) converges pointwise to zero for $\varepsilon, \varepsilon^{\prime} \searrow 0$. By a), we have $K_{\varepsilon}(x, \cdot, \mu) \in L^{1}\left(\mathbb{R}^{n}, L(E)\right)$ with a dominating function independent of $\varepsilon$, and by dominated convergence we see that for fixed $(x, y, \mu) \in \mathbb{R}^{2 n} \times\left[\omega_{0}, \infty\right)$ we get $\left\|K_{\varepsilon}(x, y, \mu)-K_{\varepsilon^{\prime}}(x, y, \mu)\right\|_{L(E)} \rightarrow 0\left(\varepsilon, \varepsilon^{\prime} \searrow 0\right)$. Therefore there exists a strongly measurable function $K: \mathbb{R}^{2 n} \times\left[\omega_{0}, \infty\right) \rightarrow L(E)$ such that $K_{\varepsilon} \rightarrow K(\varepsilon \searrow 0)$ pointwise. By dominated convergence again, inequality (3) holds for $K(x, \cdot, \mu)$ instead of $K_{\varepsilon}(x, \cdot, \mu)$.

Theorem 3.3. Let $m>0,1 \leq p<\infty, r, k, \nu \in \mathbb{N}_{0}$ with $r \geq \rho_{n,-m}+k+1, \nu \geq \rho_{n}$, and $\omega_{0}>0$. Let $b \in S^{-m, \nu, r}$. Then $b(y, D, \mu) \in L\left(W_{p}^{k}\left(\mathbb{R}^{n}, E\right)\right)$ and we have

$$
\|b(y, D, \mu)\|_{L\left(W_{p}^{k}\left(\mathbb{R}^{n}, E\right)\right)} \leq C \mu^{-m}|b|_{-m}^{(\nu, r)} \quad\left(\mu \in\left[\omega_{0}, \infty\right)\right)
$$

with a constant $C$ independent of $b$ and $\mu$.

Proof. (i) Consider first the case $k=0$. For $u \in \mathscr{S}\left(\mathbb{R}^{n}, E\right)$ we have

$$
\begin{aligned}
(b(y, D, \mu) u)(x) & =\mathrm{Os}-\iint_{\mathbb{R}^{2 n}} e^{i \xi \cdot y} b(x-y, \xi, \mu) u(x-y) d(\xi, y) \\
& =\lim _{\varepsilon \searrow 0} \int_{\mathbb{R}^{n}} \int_{\mathbb{R}^{n}} e^{i \xi \cdot y} \chi_{\varepsilon}(\xi, y) b(x-y, \xi, \mu) u(x-y) d \xi d y \\
& =\lim _{\varepsilon \searrow 0} \int_{\mathbb{R}^{n}} K_{\varepsilon}(x, y, \mu) u(x-y) d y
\end{aligned}
$$

with $K_{\varepsilon}(x, y, \mu):=\int_{\mathbb{R}^{n}} e^{i \xi \cdot y} \chi_{\varepsilon}(\xi, y) b(x-y, \xi, \mu) d \xi$. By Lemma 3.2, $K_{\varepsilon}(x, \cdot, \mu) \in$ $L^{1}\left(\mathbb{R}^{n}, L(E)\right)$ with $\varepsilon$-independent dominating function, and $K_{\varepsilon}$ converges pointwise 
to a strongly measurable function $K$ for $\varepsilon \searrow 0$. By the dominated convergence theorem, we have

$$
(b(y, D, \mu) u)(x)=\int_{\mathbb{R}^{n}} K(x, y, \mu) u(x-y) d y=[K(x, \cdot, \mu) * u](x)
$$

where $*$ stands for the standard convolution. Because of the $L^{1}$-estimate of $K$ in Lemma 3.2, we obtain for $\mu \in\left[\omega_{0}, \infty\right)$

$$
\begin{aligned}
\|b(y, D, \mu) u\|_{L^{p}\left(\mathbb{R}^{n}, E\right)} & \leq C\|K(x, \cdot, \mu)\|_{L^{1}\left(\mathbb{R}^{n}, L(E)\right)}\|u\|_{L^{p}\left(\mathbb{R}^{n}, E\right)} \\
& \leq C \mu^{-m}|b|_{-m}^{(\nu, r)}\|u\|_{L^{p}\left(\mathbb{R}^{n}, E\right) .}
\end{aligned}
$$

(ii) Now let $k \in \mathbb{N}$. By Lemma A.5, for $\beta \in \mathbb{N}_{0}^{n}$ with $|\beta| \leq k$ we write

$$
\begin{array}{r}
\left(\partial_{x}^{\beta}[b(y, D, \mu) u]\right)(x)=\mathrm{Os}-\iint_{\mathbb{R}^{2 n}} e^{i \xi \cdot y} \partial_{x}^{\beta}[b(x-y, \xi, \mu) u(x-y)] d(\xi, y) \\
=\sum_{\beta^{\prime} \leq \beta} c_{\beta \beta^{\prime}} \mathrm{Os}-\iint_{\mathbb{R}^{2 n}} e^{i \xi \cdot y}\left(\partial_{x}^{\beta^{\prime}} b\right)(x-y, \xi, \mu)\left(\partial_{x}^{\beta-\beta^{\prime}} u\right)(x-y) d(\xi, y) .
\end{array}
$$

From $\partial_{x}^{\beta^{\prime}} b \in S^{m, \nu, r-\left|\beta^{\prime}\right|}$ and part (i) of the proof we obtain

$$
\begin{aligned}
\left\|\partial_{x}^{\beta}[b(y, D, \mu) u]\right\|_{L^{p}\left(\mathbb{R}^{n}, E\right)} & \leq C \mu^{-m}|b|_{-m}^{(\nu, r)} \sum_{|\gamma| \leq k}\left\|\partial^{\gamma} u\right\|_{L^{p}\left(\mathbb{R}^{n}, E\right)} \\
& \leq C \mu^{-m}|b|_{-m}^{(\nu, r)}\|u\|_{W_{p}^{k}\left(\mathbb{R}^{n}, E\right) .}
\end{aligned}
$$

Remark 3.4. The last result shows that the mapping $b \mapsto b(y, D, \mu), S^{-m, \nu, r} \rightarrow$ $L\left(W_{p}^{k}\left(\mathbb{R}^{n}, E\right)\right)$ is continuous with norm not greater than a constant times $\mu^{-m}$ for $\mu \in\left[\omega_{0}, \infty\right)$.

Corollary 3.5. In the situation of Lemma 3.1, we have a $a^{\#}(y, D, \mu) \in L\left(W_{p}^{k}\left(\mathbb{R}^{n}, E\right)\right)$ and

$$
\sup _{a \in \mathcal{A}, \mu \geq 0}\left\|a^{\#}(y, D, \mu)\right\|_{L\left(W_{p}^{k}\left(\mathbb{R}^{n}, E\right)\right)}<\infty
$$

for all $k \in \mathbb{N}_{0}$ with $r \geq \rho_{n,-m}+k+1$.

Proof. This follows for $\mu \geq \omega_{0}$ immediately from Lemma 3.1 and Remark 3.4 while for $\mu \leq \omega_{0}$ we have $a^{\#}(y, D, \mu)=0$ for all $a \in \mathcal{A}$.

\section{Generation of an analytic semigroup}

We will need the following slight generalization of Lemma 3.2.

Lemma 4.1. Let $m>0, m_{1}, m_{2} \in \mathbb{R}$ with $m_{1}+m_{2}=-m, \nu, r \in \mathbb{N}_{0}$ with $\nu \geq n+1$, $\omega_{0}>0$. Let $b_{1} \in S^{m_{1}, \nu, r}$ and $b_{2} \in S^{m_{2}, \nu, r}$. For $\varepsilon \in(0,1)$ define

$$
K_{\varepsilon}(x, y, \mu):=\int_{\mathbb{R}^{n}} e^{i \xi \cdot y} \chi_{\varepsilon}(\xi, y) b(x, y, \xi, \mu) d \xi
$$

with $b(x, y, \xi, \mu):=\int_{0}^{1} b_{1}(x-\tau y, \xi, \mu) b_{2}(x-y, \xi, \mu) d \tau$. Then the assertions of Lemma 3.2 hold with $|b|_{-m}^{(\nu, r)}$ being replaced by $\left|b_{1}\right|_{m_{1}}^{(\nu, r)}\left|b_{2}\right|_{m_{2}}^{(\nu, r)}$. 
Proof. In the proof of Lemma 3.2, we replace inequality (4) by

$$
\left\|D_{\xi}^{\beta}[b(x, y, \mu \xi, \mu)]\right\|_{L(E)} \leq \mu^{|\beta|}\left|b_{1}\right|_{m_{1}}^{(\nu, r)}\left|b_{2}\right|_{m_{2}}^{(\nu, r)}\langle\mu \xi, \mu\rangle^{-m-|\beta|} .
$$

This can be seen by

$$
\begin{aligned}
& \left\|D_{\xi}^{\beta}[b(x, y, \mu \xi, \mu)]\right\|_{L(E)} \\
& \quad \leq \mu^{|\beta|} \sum_{\beta^{\prime} \leq \beta} c_{\beta \beta^{\prime}}\left\|\int_{0}^{1}\left(D_{\xi}^{\beta^{\prime}} b_{1}\right)(x-\tau y, \mu \xi, \mu)\left(D_{\xi}^{\beta-\beta^{\prime}} b_{2}\right)(x-y, \mu \xi, \mu) d \tau\right\|_{L(E)} \\
& \quad \leq \mu^{|\beta|} \sum_{\beta^{\prime} \leq \beta} c_{\beta \beta^{\prime}}\left|b_{1}\right|_{m_{1}}^{(\nu, r)}\langle\mu \xi, \mu\rangle^{m_{1}-\left|\beta^{\prime}\right|}\left|b_{2}\right|_{m_{2}}^{(\nu, r)}\langle\mu \xi, \mu\rangle^{m_{2}-\left(|\beta|-\left|\beta^{\prime}\right|\right)} \\
& \quad \leq \mu^{|\beta|}\left|b_{1}\right|_{m_{1}}^{(\nu, r)}\left|b_{2}\right|_{m_{2}}^{(\nu, r)}\langle\mu \xi, \mu\rangle^{-m-|\beta|} .
\end{aligned}
$$

With (6) instead of (4) we can follow the proof of Lemma 3.2 almost literally.

Throughout the remainder of this section, we fix $k \in \mathbb{N}_{0}, m>0,1 \leq p<\infty$, $r, \nu \in \mathbb{N}_{0}$ with $r \geq \rho_{n, m}+k+1$ and $\nu \geq n+2$. We will consider the $W_{p}^{k}\left(\mathbb{R}^{n}, E\right)$ realization $A_{k}$ of $a(x, D, \mu)$, i.e. the restriction of $a(x, D, \mu)$ to $D_{\max }\left(A_{k}\right):=\{u \in$ $\left.W_{p}^{k}\left(\mathbb{R}^{n}, E\right): a(x, D, \mu) u \in W_{p}^{k}\left(\mathbb{R}^{n}, E\right)\right\}$.

Theorem 4.2. Let $a \in S^{m, \nu, r}$ be parameter-elliptic, i.e., $a \in E_{\kappa, \omega}^{m, \nu, r}$ for some $\kappa>0$ and $\omega \geq 0$. Then there exist constants $M_{1}>0$ and $\mu_{1}>0$ such that the $W_{p}^{k}$-realization $A_{k}$ of $a(x, D, \mu)$ is invertible for $\mu \geq \mu_{1}$ and

$$
\left\|A_{k}^{-1}\right\|_{L\left(W_{p}^{k}\left(\mathbb{R}^{n}, E\right)\right)} \leq \frac{M_{1}}{1+\mu^{m}} \quad\left(\mu \geq \mu_{1}\right) .
$$

Proof. Let $\omega_{0}>\omega, \mu \geq 2 \omega_{0}$, and $u \in \mathscr{S}\left(\mathbb{R}^{n}, E\right)$. From Lemma 2.3 we know that

$$
a(x, D, \mu) a^{\#}(y, D, \mu) u=\left[a(x, \xi, \mu) a^{\#}(y, \xi, \mu)\right](D) u=(I+p(x, y, D, \mu)) u
$$

with the double symbol

$$
p(x, y, \xi, \mu):=(a(x, \xi, \mu)-a(y, \xi, \mu)) a(y, \xi, \mu)^{-1} .
$$

(i) Let $x \in \mathbb{R}^{n}$. By a Taylor expansion we obtain

$$
\begin{aligned}
& (p(x, y, D, \mu) u)(x) \\
& =\text { Os }-\iint_{\mathbb{R}^{2 n}} e^{i \xi \cdot y}(a(x, \xi, \mu)-a(x-y, \xi, \mu)) a(x-y, \xi, \mu)^{-1} u(x-y) d(\xi, y) \\
& =\text { Os }-\iint_{\mathbb{R}^{2 n}} e^{i \xi \cdot y} \sum_{j=1}^{n} y_{j} \int_{0}^{1}\left(\partial x_{j} a\right)(x-\tau y, \xi, \mu) a(x-y, \xi, \mu)^{-1} d \tau u(x-y) d(\xi, y) \\
& =\sum_{j=1}^{n} \operatorname{Os}-\iint_{\mathbb{R}^{2 n}} e^{i \xi \cdot y} \int_{0}^{1} D_{\xi_{j}}\left[\left(\partial x_{j} a\right)(x-\tau y, \xi, \mu) a(x-y, \xi, \mu)^{-1}\right] d \tau u(x-y) d(\xi, y) \\
& =\sum_{j=1}^{n} \lim _{\varepsilon \searrow 0} \int_{\mathbb{R}^{n}}\left[K_{\varepsilon}^{(j, 1)}(x, y, \mu)+K_{\varepsilon}^{(j, 2)}(x, y, \mu)\right] u(x-y) d y
\end{aligned}
$$

with

$$
K_{\varepsilon}^{(j, m)}(x, y, \mu):=\int_{\mathbb{R}^{n}} e^{i \xi \cdot y} \chi_{\varepsilon}(\xi, y) b^{(j, m)}(x, y, \xi, \mu) d \xi,
$$




$$
\begin{aligned}
b^{(j, 1)}(x, y, \xi, \mu) & :=\int_{0}^{1}\left(D_{\xi_{j}} \partial_{x_{j}} a\right)(x-\tau y, \xi, \mu) a(x-y, \xi, \mu)^{-1} d \tau, \\
b^{(j, 2)}(x, y, \xi, \mu) & :=\int_{0}^{1}\left(\partial_{x_{j}} a\right)(x-\tau y, \xi, \mu) D_{\xi_{j}}\left[a(x-y, \xi, \mu)^{-1}\right] d \tau .
\end{aligned}
$$

We have $D_{\xi_{j}} \partial_{x_{j}} a \in S^{m-1, r-1, \nu-1}$ and $D_{\xi_{j}} a^{-1} \in S^{-m-1, r, \nu-1}$. By Lemma 4.1 we obtain for $j=1, \ldots, n$ and $m=1,2$ the existence of a strongly measurable function $K^{(j, m)}$ with $K_{\varepsilon}^{(j, m)} \rightarrow K^{(j, m)}(\varepsilon \searrow 0)$ pointwise and

$$
\left\|K^{(j, m)}(x, \cdot, \mu)\right\|_{L^{1}\left(\mathbb{R}^{n}, L(E)\right)} \leq C \mu^{-1}|a|_{m}^{(\nu, r)}\left|a^{\#}\right|_{-m}^{(\nu, r)}
$$

for $\mu \geq 2 \omega_{0}$. Therefore,

$$
\begin{aligned}
\|p(x, y, D, \mu) u\|_{L^{p}\left(\mathbb{R}^{n}, E\right)} & \leq \sum_{j=1}^{n} \sum_{m=1}^{2}\left\|\left[K^{(j, m)}(x, \cdot, \mu) * u\right]\right\|_{L^{p}\left(\mathbb{R}^{n}, E\right)} \\
& \leq C \mu^{-1}|a|_{m}^{(\nu, r)}\left|a^{\#}\right|_{-m}^{(\nu, r)}\|u\|_{L^{p}\left(\mathbb{R}^{n}, E\right)} .
\end{aligned}
$$

(ii) Now let $\beta \in \mathbb{N}_{0}^{n}$ with $|\beta| \leq k$. In the same way as in the proof of part (ii) of Theorem 3.3, we write

$$
\begin{aligned}
& \left(\partial_{x}^{\beta} p(x, y, D, \mu) u\right)(x) \\
& \quad=\sum_{\beta^{\prime} \leq \beta} c_{\beta \beta^{\prime}} \text { Os }-\iint_{\mathbb{R}^{2 n}} e^{i \xi \cdot y}\left(\partial_{x}^{\beta^{\prime}} p\right)(x, y, \xi, \mu)\left(\partial_{x}^{\beta-\beta^{\prime}} u\right)(x-y) d(\xi, y) .
\end{aligned}
$$

Replacing in (i) $p$ by $\partial_{x}^{\beta^{\prime}} p$ and $u$ by $\partial_{x}^{\beta-\beta^{\prime}} u$, we obtain

$$
\begin{aligned}
\left\|\partial_{x}^{\beta}[p(x, y, D, \mu) u]\right\|_{L^{p}\left(\mathbb{R}^{n}, E\right)} & \leq C \sum_{\beta^{\prime} \leq \beta}\left\|\left(\partial_{x}^{\beta^{\prime}} p\right)(x, y, D, \mu)\left(\partial_{x}^{\beta-\beta^{\prime}} u\right)\right\|_{L^{p}\left(\mathbb{R}^{n}, E\right)} \\
& \leq C \sum_{\beta^{\prime} \leq \beta} \mu^{-1}|a|_{m}^{(\nu, r)}\left|a^{\#}\right|_{-m}^{(\nu, r)} \sum_{|\gamma| \leq k}\left\|\partial_{x}^{\gamma} u\right\|_{L^{p}\left(\mathbb{R}^{n}, E\right)}
\end{aligned}
$$

and therefore $\|p(x, y, D, \mu) u\|_{W_{p}^{k}\left(\mathbb{R}^{n}, E\right)} \leq C \mu^{-1}|a|_{m}^{(\nu, r)}\left|a^{\#}\right|_{-m}^{(\nu, r)}\|u\|_{W_{p}^{k}\left(\mathbb{R}^{n}, E\right)}$ for $\mu \geq$ $2 \omega_{0}$ with a constant $C$ independent of $a$ and $\mu$. Taking $\mu \geq \mu_{1}$ with $\mu_{1}$ sufficiently large, we obtain $\|p(x, y, D, \mu)\|_{L\left(W_{p}^{k}\left(\mathbb{R}^{n}, E\right)\right)} \leq \frac{1}{2}$. By a Neumann series argument, we see that $I+p(x, y, D, \mu) \in L\left(W_{p}^{k}\left(\mathbb{R}^{n}, E\right)\right)$ is invertible and therefore $a(x, D, \mu) a^{\#}(y, D, \mu)(I+p(x, y, D, \mu))^{-1}=\operatorname{id}_{W_{p}^{k}\left(\mathbb{R}^{n}, E\right)}$ for $\mu \geq \mu_{1}$. This shows that $A_{k}$ is surjective.

(iii) In the same way, we see that $a^{\#}(x, D, \mu) a(y, D, \mu)=I+\tilde{p}(x, y, D, \mu)$ with

$$
\tilde{p}(x, y, \xi, \mu):=\left(a(x, \xi, \mu)^{-1}-a(y, \xi, \mu)^{-1}\right) a(y, \xi, \mu),
$$

and that $\|\tilde{p}(x, y, D, \mu)\|_{L\left(W_{p}^{k}\left(\mathbb{R}^{n}, E\right)\right)} \leq \frac{1}{2}$ for sufficiently large $\mu$. Therefore, $(1+$ $\tilde{p}(x, y, D, \mu))^{-1} a^{\#}(x, D, \mu) a(y, D, \mu)=\operatorname{id}_{D_{\max }\left(A_{k}\right)}$. This shows that $A_{k}$ is injektive, and we obtain the invertibility of $A_{k}$. Moreover, we have for sufficiently large $\mu$

$$
\begin{aligned}
\left\|A_{k}^{-1}\right\|_{L\left(W_{p}^{k}\left(\mathbb{R}^{n}, E\right)\right)} & \leq\left\|a^{\#}(y, D, \mu)\right\|_{L\left(W_{k}^{p}\left(\mathbb{R}^{n}, E\right)\right)}\left\|(I+p(x, y, D, \mu))^{-1}\right\|_{L\left(W_{p}^{k}\left(\mathbb{R}^{n}, E\right)\right)} \\
& \leq \frac{2 M_{0}}{1+\mu^{m}}
\end{aligned}
$$

for $\mu \geq \mu_{1}$ with $\mu_{1}$ sufficiently large, where $M_{0}$ does not depend on $\mu$. 
In the following, we set $\Sigma_{\vartheta, R}:=\{\lambda \in \mathbb{C}:|\lambda| \geq R,|\arg \lambda| \leq \vartheta\}$ for $\vartheta \in[0, \pi)$ and $R>0$.

Corollary 4.3. Let $a \in S^{m, \nu, r}\left(\mathbb{R}_{x}^{n} \times \mathbb{R}_{\xi}^{n}, L(E)\right)$ be parabolic with constants $\kappa>0$ and $\omega \geq 0$, and let $A_{k}$ denote the $W_{p}^{k}\left(\mathbb{R}^{n}, E\right)$-realization of $a(x, D)$. Then there are constants $M_{2}>0, \vartheta \in\left(\frac{\pi}{2}, \pi\right)$ and $R>0$ such that for the resolvent set $\rho\left(-A_{k}\right)$ of $-A_{k}$ we have $\Sigma_{\vartheta, R} \subset \rho\left(-A_{k}\right)$,

$$
\left\|\left(\lambda+A_{k}\right)^{-1}\right\|_{L(E)} \leq \frac{M_{2}}{1+|\lambda|} \quad\left(\lambda \in \Sigma_{\vartheta, R}\right) .
$$

Therefore, $-A_{k}: W_{p}^{k}\left(\mathbb{R}^{n}, E\right) \supset D\left(A_{k}\right) \rightarrow W_{p}^{k}\left(\mathbb{R}^{n}, E\right)$ generates an analytic semigroup on $W_{p}^{k}\left(\mathbb{R}^{n}, E\right)$.

Proof. By Definition $2.4 \mathrm{~b}$ ), the symbol $a(x, \xi)+\mu^{m} e^{i \theta}$ is parameter-elliptic for all $\theta$ with $\theta \in \Sigma_{\frac{\pi}{2}, R}$. By a standard continuity argument, we see that the set of angles $\theta$ where parameter-ellipticity holds is open. Therefore, we may assume that the above symbol is parameter-elliptic for all $\theta \in \Sigma_{\vartheta, R}$ with $\vartheta>\frac{\pi}{2}$. Now the invertibility of $\lambda+A_{k}, \lambda=\mu^{m} e^{i \theta}$ and the estimate (7) follows directly from Theorem 4.2.

Remark 4.4. The proof of Theorem 4.2 shows that the constants $\mu_{1}$ and $M_{1}$ in Theorem 4.2 can be chosen independently of a for all $a \in \mathcal{A}$ where $\mathcal{A} \subset S^{m, \nu, r}$ is bounded and $\mathcal{A} \subset E_{\kappa, \omega}^{m, \nu, r}$ for fixed $\kappa>0$ and $\omega \geq 0$. In the same way, the constants $\vartheta, M_{2}$, and $R$ in Corollary 4.3 can be chosen independently of a for all $a \in \mathcal{A}^{\prime}$ where $\mathcal{A}^{\prime} \subset S^{m, \nu, r}\left(\mathbb{R}_{x}^{n} \times R_{\xi}^{n}, L(E)\right)$ is bounded and where all $a \in \mathcal{A}^{\prime}$ are parabolic with the same constants $\kappa>0$ and $\omega \geq 0$.

\section{An APPLiCATion}

Let $T>0, J:=[0, T]$ a closed interval in $\mathbb{R}$ and $t \in J$. In the following $t$ in $a(t, \cdot) \in S^{m, \nu, r}\left(\mathbb{R}^{2 n}, L(E)\right)$ and in $a(t, x, D)$ denotes only a parameter. Moreover we will consider in this section a family $\mathcal{A}:=\{a(t, \cdot): t \in J\} \subset E_{\kappa, \omega}^{m, \nu, r}$ for fixed $\kappa>0$ and $\omega \geq 0$, such that

$$
J \ni t \longmapsto a(t, \cdot) \in S^{m, \nu, r}\left(\mathbb{R}^{2 n}, L(E)\right)
$$

is a Hölder continuous function relative to the topology in the space of the symbols. We will use the results of the previous sections to study the existence and uniqueness of solutions for the Cauchy problem

$$
\left\{\begin{array}{c}
\partial_{t} u+A_{k}(t) u=f(t), \quad t \in J \backslash\{0\}, \\
u(0)=u_{0},
\end{array}\right.
$$

in $W_{p}^{k}\left(\mathbb{R}^{n}, E\right)$. There, $A_{k}(t), t \in J$, is the $W_{p}^{k}\left(\mathbb{R}^{n}, E\right)$-realization of $a(t, x, D)$ and $f:[0, T] \rightarrow W_{p}^{k}\left(\mathbb{R}^{n}, E\right)$ is a given Hölder continuous function. A function

$$
\left.\left.u \in C^{1}(] 0, T\right], E\right) \cap C([0, T], E)
$$

is called classical solution of (8) in $[0, T]$, if $u(t) \in D\left(A_{k}(t)\right)$ and $u^{\prime}(t)+A_{k}(t) u(t)=$ $f(t)$ for all $t \in] 0, T]$, and $u(0)=u_{0}$. More precisely, we will use Corollary 4.3, Theorem 2.5.1 of Chapter IV in [Am95] and Lemma 5.2 to obtain results of existence and uniqueness of solutions of the Cauchy problem (8) in $W_{p}^{k}\left(\mathbb{R}^{n}, E\right)$ if $1 \leq p<\infty$. In the following $\stackrel{\circ}{B}_{p, q}^{s}\left(\mathbb{R}^{n}, E\right), s \in \mathbb{R}, p, q \in[1, \infty]$, denote the $E$-valued homogeneous Besov spaces of order $s$ and parameters $p$ and $q$ (see [Am97] for their definition and 
properties).

Lemma 5.1. Let $m \in \mathbb{R}, \nu, r \in \mathbb{N}$ with $\nu \geq 2 n+1$ and $a \in S^{m, \nu, r}\left(\mathbb{R}^{2 n}, L(E)\right)$. Then

$$
a(x, D): \stackrel{\circ}{B}_{p, q}^{s+m}\left(\mathbb{R}^{n}, E\right) \rightarrow \stackrel{\circ}{B}_{p, q}^{s}\left(\mathbb{R}^{n}, E\right)
$$

is a bounded and linear map for all $p, q \in[1, \infty]$ and all $s \in \mathbb{R}$ with $0 \leq s<r$. Moreover it holds the following estimate:

$$
\|a(x, D)\|_{L\left(\stackrel{\leftrightarrow}{B}_{p, q}^{s+m}\left(\mathbb{R}^{n}, E\right), \stackrel{B}{B}_{p, q}^{s}\left(\mathbb{R}^{n}, E\right)\right)} \leq c|a|_{m}^{(2 n+1, r)} .
$$

Proof. See [Ki01], Theorem 3.5.

Lemma 5.2. Let $m \in \mathbb{R}, \nu \geq 2 n+1, r \geq \rho_{n, m}+k+1, p \in[1, \infty)$ and $\mathcal{A}:=$ $\{a(t, \cdot) ; t \in J\} \subset S^{m, \nu, r}$ with $A_{k}(t)$ the $W_{p}^{k}\left(\mathbb{R}^{n}, E\right)$-realization of $a(t, x, D)$. Then

$$
\stackrel{\circ}{B}_{p, 1}^{k+m}\left(\mathbb{R}^{n}, E\right) \hookrightarrow D\left(A_{k}(t)\right) \stackrel{d}{\hookrightarrow} W_{p}^{k}\left(\mathbb{R}^{n}, E\right) .
$$

Proof. It's clear that $D\left(A_{k}(t)\right) \hookrightarrow W_{p}^{k}\left(\mathbb{R}^{n}, E\right)\left(D\left(A_{k}(t)\right)\right.$ endowed with the graph norm) and it's also known that

$$
S\left(\mathbb{R}^{n}, E\right) \hookrightarrow \stackrel{\circ}{B}_{p, 1}^{k+m}\left(\mathbb{R}^{n}, E\right) \hookrightarrow \stackrel{\circ}{B}_{p, 1}^{k}\left(\mathbb{R}^{n}, E\right) \hookrightarrow W_{p}^{k}\left(\mathbb{R}^{n}, E\right), \quad 1 \leq p<\infty .
$$

From this and Lemma 5.1 it follows that $u, a(t, x, D) u \in W_{p}^{k}\left(\mathbb{R}^{n}, E\right)$ whenever $u \in S\left(\mathbb{R}^{n}, E\right)$. Therefore $S\left(\mathbb{R}^{n}, E\right) \subset D\left(A_{k}(t)\right)$. But $S\left(\mathbb{R}^{n}, E\right) \stackrel{d}{\hookrightarrow} W_{p}^{k}\left(\mathbb{R}^{n}, E\right)$ for $1 \leq p<\infty$ and then we have $D\left(A_{k}(t)\right) \stackrel{d}{\hookrightarrow} W_{p}^{k}\left(\mathbb{R}^{n}, E\right)$.

On the other hand, (10) and Lemma 5.1 imply that $\stackrel{\circ}{B}_{p, 1}^{k+m}\left(\mathbb{R}^{n}, E\right) \subset D\left(A_{k}(t)\right)$ and

$$
\begin{aligned}
\|u\|_{D\left(A_{k}(t)\right)} & =\|u\|_{W_{p}^{k}\left(\mathbb{R}^{n}, E\right)}+\|a(t, x, D) u\|_{W_{p}^{k}\left(\mathbb{R}^{n}, E\right)} \\
& \leq c_{1}\|u\|_{\dot{B}_{p, 1}^{k+m}\left(\mathbb{R}^{n}, E\right)}+c_{2}\|a(t, x, D) u\|_{\dot{B}_{p, 1}^{k}\left(\mathbb{R}^{n}, E\right)} \\
& \leq c_{1}\|u\|_{\dot{B}_{p, 1}^{k+m}\left(\mathbb{R}^{n}, E\right)}+c_{3}\|u\|_{\dot{B}_{p, 1}^{k+m}\left(\mathbb{R}^{n}, E\right)} \\
& =c\|u\|_{\dot{B}_{p, 1}^{k+m}\left(\mathbb{R}^{n}, E\right)} .
\end{aligned}
$$

So we have $\stackrel{\circ}{B}_{p, 1}^{k+m}\left(\mathbb{R}^{n}, E\right) \hookrightarrow D\left(A_{k}(t)\right)$.

Theorem 5.3. Let $k \in \mathbb{N}_{0}, \alpha, m \in \mathbb{R}^{+}$with $0<\alpha<1, p \in[1, \infty), \nu \geq 2 n+1$ and $r \geq \rho_{n, m}+k+1$. Furthermore for fixed $\kappa>0$ and $\omega \geq 0$, let $\mathcal{A}:=\{a(t, \cdot) ; t \in$ $J\} \subset E_{\kappa, \omega}^{m, \nu, r}$ be a family of parabolic symbols, such that

$$
t \longmapsto a(t, \cdot) \in C^{\alpha}\left(J, S^{m, \nu, r}\left(\mathbb{R}^{2 n}, L(E)\right)\right)
$$

and such that there is a constant $M$ with

$$
\left\|\left(\lambda+A_{k}(t)\right)^{-1}\right\|_{L\left(\dot{B}_{p, \infty}^{k}\left(\mathbb{R}^{n}, E\right), \dot{B}_{p, \infty}^{k+m}\left(\mathbb{R}^{n}, E\right)\right)} \leq M,
$$

for all $t \in J$ and $\lambda$ in the sector $\Sigma_{\vartheta, R}$ of Corollary 4.3. There $A_{k}(t)$ is the $W_{p}^{k}\left(\mathbb{R}^{n}, E\right)$-realization of a $(t, x, D)$.

If $u_{0} \in W_{p}^{k}\left(\mathbb{R}^{n}, E\right)$ and $f \in C^{\sigma}\left(J, W_{p}^{k}\left(\mathbb{R}^{n}, E\right)\right)$ for some $\left.\sigma \in\right] 0,1[$, then the Cauchy problem (8) has a unique solution

$$
u \in C\left(J, W_{p}^{k}\left(\mathbb{R}^{n}, E\right)\right) \cap C^{1}\left(J \backslash\{0\}, W_{p}^{k}\left(\mathbb{R}^{n}, E\right)\right) .
$$


Proof. First we claim that $D\left(A_{k}(t)\right) \hookrightarrow \stackrel{\circ}{B}_{p, \infty}^{k+m}\left(\mathbb{R}^{n}, E\right)$. In fact, if $u \in D\left(A_{k}(t)\right)$, then $u, a(t, x, D) u \in W_{p}^{k}\left(\mathbb{R}^{n}, E\right) \subset \stackrel{\circ}{B}_{p, \infty}^{k}\left(\mathbb{R}^{n}, E\right)$. For $\lambda_{0} \in \Sigma_{\vartheta, R}$ fixed and $v:=$ $\left(\lambda_{0}+A_{k}(t)\right) u \in \stackrel{\circ}{B}_{p, \infty}^{k}\left(\mathbb{R}^{n}, E\right),(12)$ implies that $u \in \stackrel{\circ}{B}_{p, \infty}^{k+m}\left(\mathbb{R}^{n}, E\right)$ and

$$
\begin{aligned}
\|u\|_{\stackrel{\circ}{B}_{p, \infty}^{k+m}\left(\mathbb{R}^{n}, E\right)} & \leq M\|v\|_{\stackrel{B}{p}_{p, \infty}^{k}\left(\mathbb{R}^{n}, E\right)} \\
& \leq \tilde{c}\|v\|_{W_{p}^{k}\left(\mathbb{R}^{n}, E\right)} \\
& \leq \tilde{c}\left(\left|\lambda_{0}\right|\|u\|_{W_{p}^{k}\left(\mathbb{R}^{n}, E\right)}+\left\|A_{k}(t) u\right\|_{W_{p}^{k}\left(\mathbb{R}^{n}, E\right)}\right) \\
& \leq c\|u\|_{D\left(A_{k}(t)\right)},
\end{aligned}
$$

i.e. $D\left(A_{k}(t)\right) \hookrightarrow \stackrel{\circ}{B}_{p, \infty}^{k+m}\left(\mathbb{R}^{n}, E\right)$. From this, (9), Corollary 4.3 and Theorem 2.5 .1 of Chapter IV in [Am95], we obtain the desired result in similar way to the proof of Theorem 4.3 in [BDH12].

\section{Appendix A. Appendix: Oscillatory integrals}

For the definition of pseudo-differential operators we needed the theory of (vectorvalued) oscillatory integrals. Therefore, we summarize below some definitions and results which can be found in [Ba09] and [Ku81].

Definition A.1. Let $a: \mathbb{R}^{2 n} \rightarrow E$ be a strongly measurable function. We say that $a$ is integrable in the oscillatory sense if for each $\chi \in \mathscr{S}\left(\mathbb{R}^{2 n}\right)$ with $\chi(0,0)=1$ the limit

$$
\text { Os }-\iint_{\mathbb{R}^{2 n}} e^{-i \xi \cdot y} a(\xi, y) d(\xi, y):=\lim _{\varepsilon \searrow 0} \iint_{\mathbb{R}^{2 n}} e^{-i \xi \cdot y} \chi(\varepsilon \xi, \varepsilon y) a(\xi, y) d(\xi, y)
$$

exists and does not depend on the choice of $\chi$.

Definition A.2. Let $m \in \mathbb{R}, \tau \in[0, \infty), \nu, \rho \in \mathbb{N}_{0} \cup\{\infty\}$. Then the space $\mathcal{A}_{\tau}^{(m, \nu, \rho)}\left(\mathbb{R}_{\xi}^{n} \times \mathbb{R}_{y}^{n}, E\right)$ of amplitude functions consists of all $a: \mathbb{R}_{\xi}^{n} \times \mathbb{R}_{y}^{n} \rightarrow E$ for which all derivatives $\partial_{\xi}^{\alpha} \partial_{y}^{\beta}$ a with $|\alpha| \leq \nu,|\beta| \leq \rho$ are continuous and for which all norms $|a|_{m, \ell, \ell^{\prime}}$ with $\ell, \ell^{\prime} \in \mathbb{N}_{0}, \ell \leq \nu, \ell^{\prime} \leq \rho$ are finite. Here

$$
|a|_{m, \ell, \ell^{\prime}}:=\max _{|\alpha| \leq \ell|\beta| \leq \ell^{\prime}} \sup _{\xi \in \mathbb{R}^{n}} \sup _{y \in \mathbb{R}^{n}}\langle\xi\rangle^{-m-|\beta|}\langle y\rangle^{-\tau}\left\|\partial_{\xi}^{\alpha} \partial_{y}^{\beta} a(\xi, y)\right\|_{E}
$$

Lemma A.3. For $\chi \in \mathscr{S}\left(\mathbb{R}^{n}\right)$ with $\chi(0)=1$ the following assertions hold.

(i) $\chi(\varepsilon x) \rightarrow 1(\varepsilon \searrow 0)$ uniformly on all compact subsets of $\mathbb{R}^{n}$,

(ii) $\partial_{x}^{\alpha} \chi(\varepsilon x) \rightarrow 0(\varepsilon \searrow 0)$ uniformly on $\mathbb{R}^{n}$ for all $\alpha \in \mathbb{N}_{0}^{n} \backslash\{0\}$,

(iii) for all $\alpha \in \mathbb{N}_{0}^{n}$ there exists $c_{\alpha}>0$ such that for all $\varepsilon \in(0,1)$ we have

$$
\left|\partial_{x}^{\alpha} \chi(\varepsilon x)\right| \leq c_{\alpha} \varepsilon^{\sigma}\langle x\rangle^{-(|\alpha|-\sigma)} \quad\left(\sigma \in[0,|\alpha|], x \in \mathbb{R}^{n}\right) .
$$

Proof. See [Ku81], Lemma 6.3.

Lemma A.4. Let $m \in \mathbb{R}, \tau \in[0, \infty), \ell, \ell^{\prime} \in \mathbb{N}$ with $n+\tau<2 \ell, m+n<2 \ell^{\prime}$. Let furthermore $\nu, \rho \in \mathbb{N}_{0} \cup\{\infty\}$ with $2 \ell \leq \nu, 2 \ell^{\prime} \leq \rho$. Then for $a \in \mathcal{A}_{\tau}^{(m, \nu, \rho)}\left(\mathbb{R}_{\xi}^{n} \times \mathbb{R}_{y}^{n}, E\right)$ the oscillatory integral Os $-\iint_{\mathbb{R}^{2 n}} e^{-i \xi \cdot y} a(\xi, y) đ(\xi, y)$ exists, and

$$
\left\|\mathrm{Os}-\iint_{\mathbb{R}^{2 n}} e^{-i \xi \cdot y} a(\xi, y) d(\xi, y)\right\|_{E} \leq c|a|_{m, 2 \ell, 2 \ell^{\prime}}
$$


holds with a constant $c$ not depending on a.

Proof. See [Ba09], Satz 1.3.1.

Lemma A.5. With $m, \tau, \nu, \rho, \ell$, and $\ell^{\prime}$ as in Lemma A.4, let $N \in \mathbb{N}_{0}$ and let $a: R_{x}^{n} \times \mathbb{R}_{\xi}^{n} \times \mathbb{R}_{y}^{n} \rightarrow E$ be a function with $\partial_{x}^{\gamma} a(x, \cdot, \cdot) \in A_{\tau}^{(m, \nu, \rho)}\left(\mathbb{R}_{\xi}^{n}, \mathbb{R}_{y}^{n}, E\right)$ for all $x \in \mathbb{R}^{n}$ and $|\gamma| \leq N$. Suppose further that for every $x \in \mathbb{R}^{n}$ there is a neighbourhood $U_{x}$ of $x$ such that the set $\left\{\left(\partial_{x}^{\gamma} a\right)\left(x^{\prime}, \cdot, \cdot\right): x^{\prime} \in U_{x}\right\}$ is bounded in $A_{\tau}^{(m, \nu, \rho)}\left(\mathbb{R}_{\xi}^{n}, \mathbb{R}_{y}^{n}, E\right)$. Then for all $|\gamma| \leq N$ we have

$$
\partial_{x}^{\gamma}\left[\mathrm{Os}-\iint_{\mathbb{R}^{2 n}} e^{-i \xi \cdot y} a(x, \xi, y) d(\xi, y)\right]=\mathrm{Os}-\iint_{\mathbb{R}^{2 n}} e^{-i \xi \cdot y} \partial_{x}^{\gamma} a(x, \xi, y) d(\xi, y) .
$$

Proof. See [Ba09], Satz 1.3.3.

\section{REFERENCES}

[Am95] H. Amann: Linear and quasilinear parabolic problems, Vol. I. Birkhäuser Verlag, Basel (1995).

[Am97] H. Amann: Operator-valued fourier multipliers, vector-valued besov spaces, and applications. Math. Nachr. 186 (1997), 5-56.

[Am00] H. Amann Coagulation-Fragmentation Processes, Arch. Rot. Mech. Anal. 151, 339-366 (2000).

[Am01] H. Amann, Elliptic Operators with Infinite Dimensional State Spaces, J. Evo. Equ. 1, 143-188 (2001).

[Ba09] B. Barraza Martínez, Pseudodifferentialoperatoren mit nichtregulären banachraumwertigen Symbolen, Dissertation, Johannes Gutenberg-Universität Mainz, (2009).

[BDH12] B. Barraza Martínez, R. Denk, J. Hernández Monzón, Analytic semigroups of pseudodifferential operators on vector-valued Sobolev spaces, Preprint Konstanzer Schriften in Mathematik, Nr. 303, Mai 2012.

[DHP03] R. Denk, M. Hieber, J. Prüss, $\mathcal{R}$-Boundedness, Fourier multipliers and problems of elliptic and parabolic type, Mem. Amer. Math. Soc. 166 (2003).

[DK07] R. Denk, T. Krainer, $\mathcal{R}$-boundedness, pseudodifferential operators, and maximal regularity for some classes of partial differential operators, Manuscripta Math. 124, 319-342 (2007).

[GW03] M. Girardi and L. Weis, Operator-Valued Fourier Multiplier Theorems on Besov Spaces, Math. Nachr. 251, 34-51 (2003).

[Ki01] Chr. Kiehn, Analytic Semigroup of Pseudodifferential Operators on $L_{p}\left(\mathbb{R}^{n}, E\right)$, Preprint Reihe des Fachbereichs Mathematik Nr 10, Johannes Gutenberg-Universität, (2001).

[Ki03] Chr. Kiehn, Analytic Semigroups of Pseudodifferential Operators on Vector-Valued Function Spaces, Shaker Verlag, Aachen (2003).

[Ku81] H. Kumano-go, Pseudo-Differential Operators, MIT Press, Cambridge, MA (1981).

[KW04] P. C. Kunstmann, L. Weis, Maximal $L_{p}$-regularity for parabolic equations, Fourier multiplier theorems and $H^{\infty}$-functional calculus, in Da Prato et al., editor, "Functional Analytic Methods for Evolution equations", Lecture Notes in Math. 1855, Springer, 2004 .

[NS11] T. Nau, J. Saal, $\mathcal{R}$-sectoriality of cylindrical boundary value problems, in J. Escher et al., editor, "Parabolic Problems: The Herbert Amann Festschrift", Progr. Nonlinear Differential Equations Appl. 80, Birkhuser/Springer, 479-506 (2011).

[NNH02] A. Noll, R. Haller, and H. Heck, Mikhlin's Theorem for Operator-Valued Multipliers in $n$ Variables, Math. Nach. 244, 110-130 (2002).

[PS06] P. Portal and Ž. Štrkalj, Pseudodifferential operators on Bochner Spaces and an application, Math. Z., 253, 805-819 (2006).

[We01] L. Weis, Operator-Valued Fourier Multiplier Theorems and Maximal $L_{p}$-Regularity, Math. Ann. 319, 735-758 (2001). 
Universidad del Norte, Departamento de Matemáticas, Km 5 Via a Puerto Colombia, Barranquilla, Colombia

E-mail address: bbarraza@uninorte.edu.co

Universität Konstanz, Fachbereich Mathematik, 78457 Konstanz, Germany

E-mail address: robert.denk@uni-konstanz.de

Universidad del Norte, Departamento de Matemáticas, Km 5 Via a Puerto Colombia, Barranquilla, Colombia

E-mail address: jahernan@uninorte.edu.co 